\title{
7 O CUIDADO ESPIRITUAL REALIZADO PELA ENFERMAGEM NA UNIDADE DE TERAPIA INTENSIVA' ${ }^{1}$

\author{
| Rosa Volpato²; Marislei Brasileiro; Angelica Gonçalves'; Erika Ramirez ${ }^{5}$; Gustavo Volpato ; Alisseia Lemes7 Tatiete Schonholzer ${ }^{8} \mid$
}

\begin{abstract}
CONTEXTO: Esta revisão busca responder como a enfermagem tem abordado a espiritualidade no processo de cuidar na Unidade de Terapia Intensiva (UTI), contribuindo na compreensão das dificuldades e fatores que possam melhorar a atuação destes profissionais neste ambiente de ambivalência entre a vida e a morte dos pacientes.

OBJETIVO: Verificar os artigos existentes na literatura sobre os cuidados espirituais prestados pela equipa de enfermagem em UTI.

MÉTODOS: Foi utilizado o método de revisão integrativa, sendo as buscas realizadas entre 2008 a 2018 nas bases de dados Medline, Lilacs, Cinahl e no portal PubMed. Foram utilizados chaves de busca com os descritores Spirituality, Nursing, Team, Intensive Care Units, Systematization of Nursing Care e suas variações em português e espanhol, com os operadores booleanos AND e/ou OR.

RESULTADOS: Foram encontrados na literatura 381 artigos, sendo que ao final do processo foram incluídos quatro artigos que descreviam os cuidados espirituais prestados pela equipa de enfermagem em UTI. Foram elencadas três categorias que tratam da temática: Processo de Enfermagem e os cuidados espirituais; b) Bem-estar espiritual; c) Desafios na assistência aos cuidados espirituais.

CONCLUSÕES: A espiritualidade dentro do processo de cuidar foi relatada em poucos estudos e de forma fragmentada. Mostrou-se importante o cuidar de si para cuidar do outro, além de aspectos relacionados ao enfrentamento de desafios. A enfermagem necessita ter um olhar mais amplo do cuidado espiritual, para que possa ofertar o cuidado sistematizado e com embasamento científico.
\end{abstract}

KEYWORDS: Espiritualidade; Enfermagem; Unidades de terapia intensiva; Processo de enfermagem

\section{RESUMEN}

\section{"El cuidado espiritual realizado por enfermería en la unidad de cuidados intensivos"}

CONTEXTO: Esta revisión busca dar respuesta a cómo la enfermería ha abordado la espiritualidad en el proceso de cuidado en la Unidad de Cuidados Intensivos (UCI), contribuyendo a la comprensión de las dificultades y factores que pueden mejorar el desempeño de estos profesionales en este ambiente ambivalente entre la vida y la muerte de los pacientes.

OBJETIVO: Verificar los artículos existentes en la literatura sobre el cuidado espiritual proporcionado por el equipo de enfermería en las UCI.

METODOLOGÍA: Se utilizó el método de revisión integradora con las búsquedas realizadas entre 2008 y 2018 en las bases de datos LILACS, MEDLINE, PubMed y Cinahl. Las claves de búsqueda se utilizaron con los descriptores Spirituality, Nursing, Team, Intensive Care Units, Systematization of Nursing Care y sus variaciones en portugués e español, com los operadores booleanos Y y/o OR.

RESULTADOS: En la literatura, se encontraron 381 artículos y, al final del proceso, se incluyeron cuatro artículos que incluían el cuidado espiritual proporcionado por el equipo de enfermería de la UCI. Se enumeraron tres categorías que abordan el tema: a) Proceso de enfermería y el cuidado espiritual; b) Bienestar espiritual; c) Retos en la asistencia del cuidado espiritual.

CONCLUSIONES: La espiritualidad dentro del proceso de atención se ha informado en pocos estudios y de forma fragmentada. Resulta importante cuidarse a sí mismo para cuidar de los demás, así como los aspectos relacionados con los desafíos que enfrenta. La enfermería necesita tener una visión más amplia del cuidado espiritual, de modo que pueda ofrecer un cuidado sistematizado y con base científica.

DESCRIPTORES: Espiritualidad; Enfermería; Unidades de cuidados intensivos; Proceso de enfermería

\section{RESUMO}

"The spiritual care carried out by nursing in the intensive care unit"

BACKGROUND: This review seeks to answer how nursing has addressed spirituality in the care process in the Intensive Care Unit (ICU), contributing to the understanding of the difficulties and factors that can improve the performance of these professionals in this ambivalent environment between patients' lives and deaths.

AIM: Verify the existing studies in the literature on spiritual care provided by the nursing team in the ICU.

METHODS: The integrative review method was used, and the searches performed between 2008 and 2018 in the databases Medline, Lilacs, Cinahl and the PubMed portal. The search keys were used with the descriptors Spirituality, Nursing, Team, Intensive Care Units, Systematization of Nursing Care and its variations in Spanish and Portuguese, using Boolean operators AND and/or OR.

RESULTS: In the literature, 381 articles were found and, at the end of the process, four articles were included that presented the spiritual care provided by the ICU nursing team. Three categories that address the issue were listed: a) Nursing process and spiritual care; b) Spiritual well-being; c) Challenges in assisting spiritual care.

CONCLUSIONS: Spirituality within the attention process has been reported in few studies and of fragmented way. It was important to take care of yourself to take care of others, as well as aspects related to the facing challenges. Nursing needs to have a broader vision of spiritual care, so that it can offer systematized care scientific basis.

\section{PALAVRAS-CHAVE: Spirituality; Nursing; Intensive care units; Nursing process \\ Submetido em 30-12-2019 \\ Aceite em 02-03-2020}


e a espiritualidade no processo de cuidar na Unidade de Terapia Intensiva: Revisão Integrativa" (2018), apresentado no Centro de Estudos de Enfermagem e Nutrição.

2 Enfermeira; Doutoranda em Enfermagem pela Universidade de São Paulo, Escola de Enfermagem, São Paulo, Brasil, rosamjvolpato@gmail.com

3 Enfermeira; Docente na Pontifícia Universidade Católica de Goiás e Centro de Estudos de Enfermagem e Nutrição, Goiânia, Brasil, marislei@cultura.trd.br

4 Enfermeira; Docente na Universidade Federal de São Carlos, Departamento de Enfermagem, São Carlos, São Paulo, Brasil, angelicamartins@ufscar.br

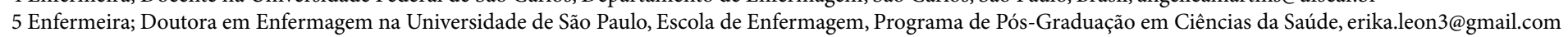
6 Biomédico; Docente na Universidade Federal de Mato Grosso, Barra do Graças, Mato Grosso, Brasil, gtvolpato@gmail.com


hotmail.com



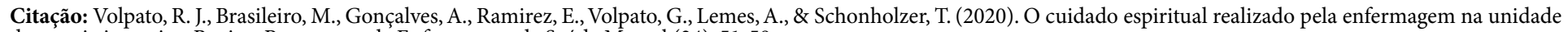
de terapia intensiva. Revista Portuguesa de Enfermagem de Saúde Mental (24), 51-58. 


\section{INTRODUÇÃO}

A espiritualidade é um termo amplo, com significado muitas vezes associado à religiosidade. A palavra espiritualidade é derivada do latim "spiritus" que tem como significado o sopro, vento ou respiração. Está ligada à subjetividade humana na busca do significado, sentido para tudo na vida, que pode ou não estar associada à religiosidade (Valente et al., 2016). Dada a sua importância, ela foi adicionada ao conceito multidimensional em saúde, juntamente com as dimensões físicas, psicológicas e sociais, pela Organização Mundial de Saúde (OMS) no final da década de 90 (Júnior et al., 2015).

A espiritualidade está associada à qualidade de vida geral dos indivíduos, enfatizando o benefício e a importância da sua inserção para o cuidado dos usuários que se encontram em diversas condições de saúde (Ienne et al., 2018); sendo também apontada no aumento da sobrevida, diminuição da prevalência de doenças e aumento do autocuidado. Já a espiritualidade vivenciada de forma negativa pode vir associada a desfechos prejudiciais à saúde (Moreira-Almeida e Lucchetti, 2016). Entre os profissionais de saúde, a espiritualidade é mencionada como alternativa/apoio para diminuir a ansiedade (Moura et al., 2018). Portanto, o exposto nos aponta que a espiritualidade faz parte do dia-a-dia da sociedade em geral, independente da crença religiosa.

A participação do profissional de enfermagem, direcionada aos aspectos espirituais, pode auxiliar no processo de cuidado centrado, integral e de qualidade a esses pacientes (Conselho Federal de Enfermagem [COFEN], 2017). Para direcionar o cuidado utiliza-se o Processo de Enfermagem (PE), sendo de suma importância para garantir uma assistência sistematizada pela equipa de enfermagem (Viana et al., 2018). Isso proporciona um cuidado efetivo, detalhado e organizado, que facilita a verificação da efetividade no tocante ao paciente e/ou ao familiar. Como os aspectos espirituais fazem parte das necessidades e preocupações dos pacientes em condições críticas de saúde e com risco iminente de morte (Braz Evangelista et al., 2016), estes sentimentos e desejos podem estar mais aflorados quando o internamento se dá na UTI. Visando responder ao nosso questionamento de como a enfermagem tem abordado a espiritualidade no processo de cuidar na UTI, esta revisão busca trazer contribuições para compreender as dificuldades e fatores que possam colaborar para melhorar a atuação destes profissionais, que vivem num ambiente que lembra muitas vezes a ambivalência entre a vida e a morte dos pacientes.
Diante do exposto, o objetivo do estudo foi verificar os artigos existentes na literatura a respeito dos cuidados espirituais prestados pela equipa de enfermagem em UTI.

\section{MÉTODOS}

Optou-se por utilizar a revisão integrativa com o propósito de agrupar e resumir resultados disponíveis em estudos, que tratam da temática sobre a enfermagem e a espiritualidade no processo de cuidar na UTI, de forma ordenada e sistemática para contribuir com a reflexão. Para tal propósito foram utilizados os seguintes passos descritos por Cooper (1984): Formulação do problema, levantamento dos dados, avaliação dos dados coletados, análise e interpretação dos resultados. As buscas por bibliografias, realizadas no período entre 2008 a 2018 nas bases indexadas de dados Medline, Lilacs, Cinahl e no portal PubMed. Foram utilizadas estratégias de busca com os descritores Spirituality, Nursing, Team, Intensive Care Units, Systematization of Nursing Care e suas variações em português e espanhol, com os operadores booleanos AND e/ou OR. Cabe ressaltar que as chaves foram utilizadas de acordo com as características de cada base de dados.

Os critérios de inclusão foram estudos que estavam disponíveis na íntegra, artigos originais, qualitativos e/ou quantitativos, nos idiomas português, espanhol ou inglês. De acordo com os critérios de inclusão, não foram considerados relatos de experiência, editoriais, cartas, artigos de opinião e de revisão, comentários, notas prévias, teses e dissertações. Os artigos selecionados (Figura 1) foram exportados para o computador e quando verificado duplicidade já eram excluídos. Posteriormente procedeu-se a leitura de títulos e resumos dos demais artigos e em seguida a leitura dos artigos na íntegra. Após essa fase, foram extraídos os principais resultados, utilizando como referência as etapas do Processo de Enfermagem (PE): a Recolha de Dados; Diagnóstico de Enfermagem; Planeamento de Enfermagem; Implementação e Avaliação) e análise de conteúdo convergente, onde se criaram categorias de análises por similaridade de conteúdo. 
Figura 1 - Fluxograma ilustrativo do processo de seleção dos artigos das bases de dados Lilacs, Medline, PubMed e Cinahl.

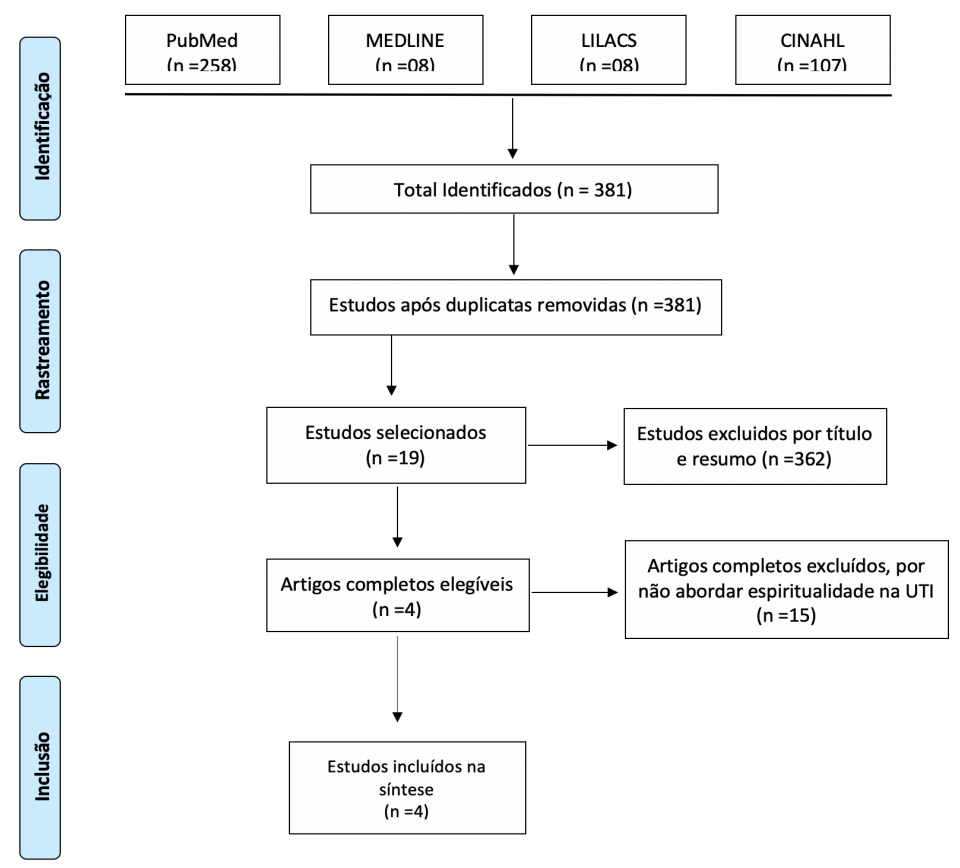

\section{RESULTADOS}

Foram encontrados na literatura 381 artigos, sendo que ao final do processo foram incluídos quatro artigos que traziam os cuidados espirituais prestados pela equipa de enfermagem em UTI (Quadro 1). Três estudos são qualitativos (dois utilizaram como técnica de pesquisa de dados a entrevista e um utilizou o método criativo sensível de Cabral) e um é quantitativo correlacional descritivo. Quanto ao país onde foram realizados os estudos, dois são do Brasil, um da Tailândia e um do Irã, sendo o ano de publicação de 2008 a 2015.

Dois dos artigos estavam indexados na base de dados LILACS, um no MEDLINE e um no Pubmed. A partir da análise dos resultados descritos nos artigos, foram elencadas as categorias principais que tratam dos cuidados espirituais prestados pela equipa de enfermagem em UTI, a saber: a) Processo de Enfermagem e os cuidados espirituais; b) Bem-estar espiritual; c) Desafios na assistência aos cuidados espirituais

Quadro 1 - Autores, ano de publicação, país de origem, objetivo, desenho do estudo, resultados e conclusão.

\begin{tabular}{|c|c|c|c|c|c|}
\hline 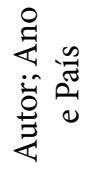 & $\frac{\pi}{0}$ & Objetivo & Desenho do estudo & Resultados & Conclusão \\
\hline 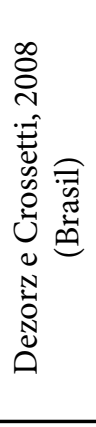 & 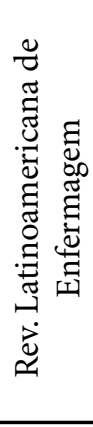 & $\begin{array}{l}\text { Compreender } \\
\text { como a espiritu- } \\
\text { alidade permeia o } \\
\text { processo de cuidar } \\
\text { de si e do outro, no } \\
\text { mundo da terapia } \\
\text { intensiva, sob o ol- } \\
\text { har das profission- } \\
\text { ais de enfermagem }\end{array}$ & $\begin{array}{l}\text { Abordagem qualitativa, } \\
\text { método criativo-sensível de } \\
\text { Cabral, que guiou a produção } \\
\text { e a análise das informações } \\
\text { em nove oficinas de arte e } \\
\text { experiências. Participaram } \\
\text { n=9 profissionais da equipa de } \\
\text { enfermagem, atuantes na UTI } \\
\text { em um hospital universitário. }\end{array}$ & $\begin{array}{l}\text { Este texto apresenta um dos temas } \\
\text { emergidos na pesquisa: a espirituali- } \\
\text { dade no cuidado de si, que foi eviden- } \\
\text { ciada nas práticas cotidianas que acon- } \\
\text { teciam por meio da oração, do contato } \\
\text { íntimo com a natureza, assim como } \\
\text { do senso de conexão com uma Força } \\
\text { Superior que propiciava tranquilidade, } \\
\text { bem-estar e fortalecimento à vida e ao } \\
\text { trabalho das cuidadoras na UTI. }\end{array}$ & $\begin{array}{l}\text { O autoconhecimento } \\
\text { revelou-se como prática } \\
\text { essencial no cuidado de } \\
\text { si para também melhor } \\
\text { cuidar do outro. }\end{array}$ \\
\hline 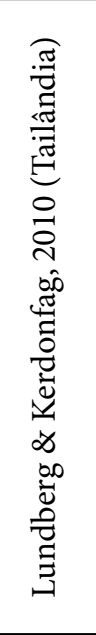 & 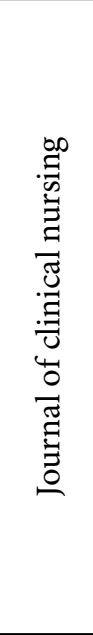 & $\begin{array}{l}\text { Explorar como } \\
\text { os enfermeiros } \\
\text { tailandeses em } \\
\text { unidades de terapia } \\
\text { intensiva de um } \\
\text { hospital universi- } \\
\text { tário em Bangkok } \\
\text { prestam cuidados } \\
\text { espirituais aos seus } \\
\text { pacientes. }\end{array}$ & $\begin{array}{l}\text { Enfermeiras } \mathrm{n}=30 \text {, } \\
\text { amostragem intencional com a } \\
\text { técnica de bola de neve. } \\
\text { Entrevistas semiestruturadas } \\
\text { com questões abertas, gra- } \\
\text { vadas, transcritas na íntegra } \\
\text { e submetidas à análise de } \\
\text { conteúdo. }\end{array}$ & $\begin{array}{l}\text { Cinco temas relacionados à prestação } \\
\text { de cuidados espirituais surgiram: dar } \\
\text { apoio mental, facilitar rituais religiosos } \\
\text { e crenças culturais, comunicar-se com } \\
\text { pacientes e familiares de pacientes, } \\
\text { avaliar as necessidades espirituais dos } \\
\text { pacientes e mostrar respeito e facilitar } \\
\text { a participação da família nos cuidados. } \\
\text { Diversas formas de melhorar o cui- } \\
\text { dado espiritual foram sugeridas pelos } \\
\text { enfermeiros. }\end{array}$ & $\begin{array}{l}\text { A espiritualidade foi uma } \\
\text { parte importante do cui- } \\
\text { dado para os enfermeiros } \\
\text { ao atender as necessi- } \\
\text { dades de seus pacientes e } \\
\text { das famílias dos pacien- } \\
\text { tes. Portanto, a educação } \\
\text { em enfermagem deve } \\
\text { melhorar a compreensão } \\
\text { e a consciência dos en- } \\
\text { fermeiros sobre questões } \\
\text { espirituais e prepará- } \\
\text { los para responder às } \\
\text { necessidades espirituais } \\
\text { humanas. }\end{array}$ \\
\hline
\end{tabular}




\begin{tabular}{|c|c|c|c|c|c|}
\hline 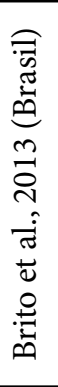 & 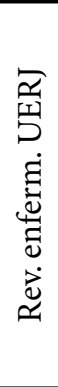 & $\begin{array}{l}\text { Investigar a } \\
\text { compreensão de } \\
\text { enfermeiros sobre } \\
\text { conceitos de espiri- } \\
\text { tualidade e de neces- } \\
\text { sidades espirituais } \\
\text { do paciente sem } \\
\text { possibilidades ter- } \\
\text { apêuticas. }\end{array}$ & $\begin{array}{l}\text { Pesquisa exploratória, com } \\
\text { abordagem qualitativa, } \mathrm{n}=7 \\
\text { enfermeiras de UTI em um } \\
\text { hospital público. } \\
\text { As entrevistas foram gravadas } \\
\text { e tratados qualitativamente } \\
\text { mediante a técnica de análise } \\
\text { de conteúdo. }\end{array}$ & $\begin{array}{l}\text { As categorias emergidas do material } \\
\text { empírico foram: espiritualidade na } \\
\text { visão de enfermeiros; e necessidades } \\
\text { espirituais dos pacientes sem possibi- } \\
\text { lidades terapêuticas: compreensão de } \\
\text { enfermeiros. }\end{array}$ & $\begin{array}{l}\text { A partir da compreensão } \\
\text { da dimensão espiritual, } \\
\text { os profissionais passam } \\
\text { a valorizá-la na prática } \\
\text { clínica, ajudando o paci- } \\
\text { ente a enfrentar melhor } \\
\text { o processo de terminali- } \\
\text { dade. }\end{array}$ \\
\hline 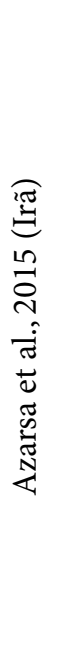 &  & $\begin{array}{l}\text { Avaliar o bem- } \\
\text { estar espiritual, a } \\
\text { atitude em relação } \\
\text { cuidado espiritual } \\
\text { e sua relação com } \\
\text { a competência do } \\
\text { cuidado espiritual } \\
\text { entre enfermeiros. }\end{array}$ & $\begin{array}{l}\text { Estudo descritivo correlacio- } \\
\text { nal, } \mathrm{n}=108 \text { enfermeiros atu- } \\
\text { antes nas UTI's, em } 2 \text { hospitais } \\
\text { do Irã. } \\
\text { Foram utilizados: formulário } \\
\text { de dados demográficos. } \\
\text { Três escalas: Escala de Bem- } \\
\text { Estar Espiritual, Espirituali- } \\
\text { dade e Escala de Avaliação de } \\
\text { Cuidados Espirituais e Escala } \\
\text { de Competência de Cuidado } \\
\text { Espiritual. Os dados foram } \\
\text { analisados pelo SPSS. }\end{array}$ & $\begin{array}{l}\text { A pontuação média do bem-estar } \\
\text { espiritual= } 94,45(14,84) \text {, a perspectiva } \\
\text { do cuidado espiritual= } 58,87(8,67) \\
\text { e a competência do cuidado espiri- } \\
\text { tual= } 98,51(15,44) \text {. Regressão linear } \\
\text { mostrou uma variação de } 0,42 \text { entre } \\
\text { as pontuações de competência no cui- } \\
\text { dado espiritual explicadas pelo bem- } \\
\text { estar espiritual (saúde religiosa, saúde } \\
\text { existencial) e aspectos da perspectiva } \\
\text { do cuidado espiritual (espirituali- } \\
\text { dade, cuidado espiritual, atendimento } \\
\text { personalizado). A competência do } \\
\text { cuidado espiritual teve uma relação } \\
\text { positiva com o bem-estar espiritual e a } \\
\text { perspectiva do cuidado espiritual. }\end{array}$ & $\begin{array}{l}\text { Devido à natureza da } \\
\text { enfermagem e im- } \\
\text { portância da interação } \\
\text { próxima enfermeiros com } \\
\text { pacientes em UTIs, maior } \\
\text { bem-estar espiritual dos } \\
\text { enfermeiros e quanto } \\
\text { maior sua atitude positiva } \\
\text { em relação ao cuidado es- } \\
\text { piritual, mais eles podem } \\
\text { fornecer cuidados aos } \\
\text { seus pacientes }\end{array}$ \\
\hline
\end{tabular}

\section{DISCUSSÃO}

\section{Processo de Enfermagem (PE) e os Cuidados Espiri- tuais}

A prática do cuidado em enfermagem diário é embasado em funções sistematizadas exercidas pelos enfermeiros. Segundo o Conselho Federal de Enfermagem (COFEN - Brasil, Resolução 358/2009), o PE é uma ferramenta de organização da assistência de enfermagem, disposta nas seguintes fases: Recolha de Dados; Diagnóstico de Enfermagem; Planeamento de Enfermagem; Implementação e; Avaliação de Enfermagem (COFEN, 2009). Isso demarca o enfermeiro como um profissional relevante e autónomo para realizar o cuidado.

Observamos que os artigos incluídos no estudo não apresentam todas as fases desse processo bem delineadas. Isso foi verificado por Viana et al. (2018), que aponta a execução fragmentada do PE devido às falhas no conhecimento científico e falta de tempo ou capacitação. Além disso, o cuidado é realizado desvinculado da sistematização da assistência, dificultando a sua percepção e continuidade deste cuidado.

Entretanto, foi possível identificar recolha de dados e diagnóstico de enfermagem nos estudos de Brito et al. (2013) e Lundberg et al. (2010). Estes autores relataram que a comunicação verbal (com o paciente ou com a família) ou não-verbal (símbolos que remetem a crenças religiosas) são os principais meios de identificação das necessidades e sofrimento espirituais dos pacientes na UTI. Ao realizar o cuidado espiritual, o enfermeiro tem que apresentar uma destreza eficaz durante a comunicação, pois a sua importância é ímpar para a identificação das necessidades espirituais e o sofrimento espiritual (Caldeira \& Timmins, 2017).

Para que ocorra a identificação adequada das necessidades dos pacientes, o enfermeiro precisa ter interesse em conhecê-lo e aplicar o conhecimento e as habilidades para realizar o PE (Sousa et al., 2018). Este é um processo constante, caracterizado pela pesquisa de informações e observação dos usuários e familiares, determinando assim, as intervenções das suas necessidades espirituais (Caldeira \& Timmins, 2017). Concernente a isso, observa-se a importância de uma minuciosa pesquisa de informações, pois há indícios de forte correlação entre diagnóstico de enfermagem e os resultados positivos das intervenções propostas, reforçando assim a necessidade de um amplo conhecimento científico na prática da enfermagem (Leoni-Scheiber et al., 2020). Após a pesquisa de informações, é realizado o planeamento e a implementação das ações de enfermagem, que dependem da elaboração de intervenções para alcançar os resultados. Neste contexto, o estudo de Lundberg et al. (2010) apresentou mecanismos para proporcionar alivio espiritual para os pacientes, sendo a gravação de orações para que os pacientes pudessem 
ouvir em diversos momentos do seu internamento, a escuta da família e dos pacientes sobre a espiritualidade, estar ao lado, tocar ou segurar a mão do paciente e o amparo as famílias. Cabe ressaltar que a morte pode ser vivenciada de maneiras distintas, e compete ao profissional a imparcialidade durante a assistência (Brasileiro e Brasileiro, 2017). Porém, a espiritualidade é uma importante ferramenta para auxiliar na resiliência nestes momentos de difícil enfrentamento (Braz Evangelista et al., 2016).

Os autores Brito et al. (2013) e Lundberg et al. (2010) enfatizam também a importância do Planeamento ser realizado por uma equipa multidisciplinar, para que os cuidados prestados contemplem as necessidades físicas, psicossociais e espirituais dos pacientes. Os profissionais que atuam na UTI apresentam competências, um alto grau de conhecimento e o comprometimento para desempenhar o apoio continuado para com a família e o paciente (Gulini et al., 2017). Os membros desta equipa devem estar atentos para o cuidado espiritual, e desta forma compartilhar informações na elaboração para o plano de cuidado de forma coletiva e não-fragmentada. A última fase do PE é a avaliação e, apesar dos estudos não trazerem claramente essa etapa, foram identificados relatos de profissionais que tiveram um retorno positivo de familiares de pacientes a respeito dos cuidados espirituais prestados pela equipa. Outros profissionais acreditam na importância do desenvolvimento de ferramentas de avaliação para as necessidades espirituais (Lundberg et al., 2010). É apontado carência de instrumentos que auxiliem na avaliação e qualificação do cuidado individualizado após sua implementação (Barros et al., 2015). Na avaliação do cuidado espiritual, Braz Evangelista et al. (2016) assinalam que 73 instrumentos apresentam este questionamento, e a maioria são voltados para o cuidado de pessoas com câncer e/ou cuidados paliativos, destacando que cerca de $10 \%$, ou seja, nove instrumentos passaram por adaptação transcultural.

As UTIs possuem características de dinâmica de trabalho diferenciadas de outros setores hospitalares (pacientes em estado críticos e altas densidades tecnológicas) e geralmente os cuidados (diagnóstico de enfermagem) acabam sendo focados nas necessidades psicobiológicas para manutenção da vida (Sousa et al., 2018). No entanto, a espiritualidade permeia o ambiente da UTI, auxiliando na aceitação do quadro da doença, diminuindo o sofrimento e trazendo conforto, tanto para a família e o paciente (Vale e Líbero, 2017). Mesmo que o cuidado espiritual, ainda hoje, não seja visto pela enfermagem como um cuidado básico em saúde.
O enfermeiro necessita entender o significado de espiritualidade para saber o que observar e questionar durante a consulta, e a aquisição de conhecimento em relação a amplitude espiritual existente, para que assim, reflita em uma assistência espiritual efetiva e que atenda às necessidades dos pacientes e familiares (Braz Evangelista et al., 2016).

\section{Bem-estar espiritual}

Os estudos trazem a concepção de bem-estar próprio para prestar cuidados aos outros Dezorzi et al., 2008; Azarsa et al., 2015). A profissão da enfermagem possui teorias, sendo uma dessas a Teoria das necessidades Humanas Básicas de Wanda Horta. Essa teoria define que o enfermeiro deve garantir cuidados em todas as suas dimensões, sendo gente que cuida de gente, não apenas como paciente e sim como ser humano (Barros et al., 2015).

Porém, muitas vezes o cuidar de si entre os profissionais de enfermagem não é algo rotineiro. Isto é evidenciado por rotinas estressantes e de sobrecarga de trabalho, com infortúnios da dor e de sofrimento, gerando cansaço físico e mental (Salimena et al., 2016). Moura et al. (2018) apontaram que 30\% dos profissionais de saúde apresentavam algum grau de ansiedade, associado à pressão exercida no trabalho, dificuldades de dormir e tratamento para ansiedade. Nesse sentido, é necessário recordar que enfermeiro faz parte de uma equipa multiprofissional, onde todos são responsáveis pela assistência espiritual e apoio aos pacientes e familiares (Caldeira \& Timmins, 2017). Esse compartilhamento da assistência pode ser o fator para diminuir a carga de trabalho sobre os enfermeiros. Os trabalhadores da saúde que atuam na UTI apresentam maior risco de desenvolver Burnout. Ho et al. (2018) também destacam a necessidade investigar quem são estes profissionais e como o cuidado espiritual pode auxiliá-los. Isso tornase essencial para melhorar a oferta do cuidado por estes indivíduos. Os estudos de Dezorzi et al. (2008) e Azarsa et al. (2015) descrevem que os hábitos de autocuidado (estar em contato com a natureza, acreditar em uma força superior, realizar práticas de orações e compartilhar com colegas suas experiências) ajudam na busca de forças para lidar com o ambiente intenso com que trabalham. Além disso, ajudam também na ampliação da sensibilidade para perceber as necessidades espirituais dos pacientes (Dezorzi et al., 2008). Os profissionais de enfermagem estão preparados para identificar tais necessidades, mas primeiramente eles precisam estar preparados para identificar as suas próprias necessidades, e assim poder realizar as ações de cuidado espiritual para com o outro (Ienne et al., 2018). 
Azarsa et al. (2015) apontam que o bem estar espiritual entre os enfermeiros está diretamente correlacionado ao aumentando da atitude positiva em relação à espiritualidade e ao cuidado espiritual na enfermaria de UTI. Corroborando com esta pesquisa, Ienne et al. (2018) mostram que enfermeiros que apresentavam maior conexão espiritual em momentos difíceis lidam melhor com o estresse, sentem-se confortados e bem no dia-dia devido à contribuição da fé. Estes fatores estão relacionados com a vontade de conversar sobre espiritualidade com o paciente.

Um fator importante é o cuidar de si para cuidar do outro, sendo representado por questões que transcendem ter saúde ou equilíbrio no ambiente de trabalho. Está ligada ao autoconhecimento, qualidade de vida, além do aumento da sensibilidade para com o outro, colocando- se na posição de sujeito e não apenas objeto (Salimena et al., 2016). Sendo assim, o bem-estar físico e espiritual do profissional de saúde capacita-o a um cuidado mais abrangente ao paciente. Portanto, a enfermagem pode auxiliar o paciente a alcançar o seu bemestar, mesmo no momento de enfermidade, através do cuidado espiritual adequado de forma individual para contemplar as suas necessidades (Caldeira \& Timmins, 2017).

\section{Desafios na assistência aos cuidados espirituais}

Uma revisão narrativa apontou que existem poucos estudos que abordam as necessidades espirituais dos pacientes nas UTIs, mesmo sendo evidente que estes pacientes e seus familiares carecem receber este cuidado. Os profissionais de saúde devem ofertar o cuidado para amparar os familiares e o indivíduos no enfrentamento das condições críticas de saúde (Ho et al., 2018). Esta situação fica clara nos relatos emergidos nos estudos de Brito et al. (2013) e Lundberg et al. (2010), que enfatizam a dificuldade ou despreparo dos profissionais para perceber as necessidades ou sofrimentos espirituais de pacientes, principalmente daqueles que estão impossibilitados de expressar verbalmente suas angústias. Cabe à equipa conscientizar-se dos desafios e buscar meios para transpor as barreiras de comunicação e permitir a humanização da assistência (Brasileiro e Brasileiro, 2017). Portanto, os enfermeiros têm que melhorar o raciocínio clínico e habilidades no conhecimento da espiritualidade, mesmo sendo subjetiva (Ienne et al., 2018). Já o estudo de Azarsa et al. (2015) mostra que os profissionais de enfermagem possuem um nível de conhecimento adequado para poder prestar cuidados espirituais.
Algumas variáveis que interferem na execução do PE são destacadas, como estruturas físicas, carga de trabalho e défice de recursos humanos. Isso pode acarretar no distanciamento na utilização do $\mathrm{PE}$, que muitas vezes não consegue ultrapassar os limites do conhecimento teórico para a prática (Barros et al., 2015). Neste sentido, outro estudo aponta que a falta de tempo, devido a uma rotina de trabalho intensiva, pode prejudicar a execução da prestação do cuidados espirituais, pois delimita ato de ouvir e observar (Lundberg et al., 2010). Outro ponto de desafio para os profissionais é a comunicação com os pacientes, em virtude das condições críticas e equipamentos que não permitem a verbalização e, sendo muitas vezes eles próprios, analfabetos, não permitindo a comunicação através da escrita. A comunicação com a família também se pode tornar um desafio pela não-aceitação da condição crítica do seu familiar, interrompendo assim a comunicação com a equipa de enfermagem (Lundberg et al., 2010). Um fator primordial na assistência seria conseguir aproximar essas famílias da assistência prestada, pois é de grande relevância para o planeamento e desenvolvimento de ações durante o cuidado realizado na UTI (Brasileiro e Brasileiro, 2017). Outro fator relatado é a formação de futuros profissionais de enfermagem, uma vez que possuem conhecimentos insuficientes a respeito dos cuidados espirituais. Uma sugestão seria a inserção de disciplinas e cursos para refinar tais cuidados (Lundberg et al., 2010; Azarsa et al., 2015). Assim se percebem que existem lacunas deste conhecimento e/ou da prática de como identificar as necessidades espirituais e de como implementar tais cuidados, sendo considerado um desafio, preocupando tanto graduandos como profissionais em atuação (da Silva et al., 2015).

Para Ienne et al. (2018) há a necessidade de inserir o tema da espiritualidade, não apenas no currículo da graduação, mas também na prática da docência, favorecendo a prática do enfermeiro de forma integral. Isto proporcionaria ao enfermeiro reconhecer as necessidades do paciente para a elaboração de diagnósticos específicos e o devido registro para a continuidade do cuidado, bem como a identificação detalhada do trabalho realizado pelo enfermeiro. Porém, segundo Timmins \& Caldeira (2017) a capacitação pode não gerar um conhecimento que abarque as complexidades da espiritualidade, limitando a eficácia do cuidado.

Os desafios apontados nos estudos merecem ter um olhar diferenciado, pois é através da identificação que podemos buscar soluções para melhorar a assistência das necessidades espirituais dentro da UTI. 
Para garantir uma assistência individualizada aos pacientes que se encontram fragilizados no ambiente de cuidados intensivos, há necessidade de aprimoramento e a inter-relação entre teoria e prática, visando garantir a compreensão de condições biopsicossociais e espirituais.

\section{CONCLUSÕES}

Diante dos resultados apresentados, conclui-se que a espiritualidade dentro do cuidado sistematizado em UTI foi identificada em poucos estudos, onde foram extraídos das falas dos profissionais apenas etapas fragmentadas do processo de enfermagem. Evidenciou-se a importância do profissional apresentar uma atitude positiva quanto à espiritualidade e auto cuidado para prestar os cuidados a outras pessoas. Alguns desafios ainda devem ser enfrentados, seja durante a formação como profissional ou quanto a situações de recursos estruturais e humanos disponíveis para a atuação no ambiente de trabalho. Portanto, foi possível desvelar o contexto representativo e atual do cuidado espiritual prestado pela enfermagem na UTI. Isso permite que a enfermagem possa ter uma visão ampla de que a espiritualidade permeia toda a forma de cuidado oferecido em diversos ambientes aos pacientes e seus familiares. Considera-se como limitação a não apresentação do nível de evidência dos estudos utilizados para compor esta revisão, o que poderia incorporar maior robustez aos achados. Todavia, como não foram encontrados muitos estudos, utilizar esses instrumentos de avaliação poderia limitar os resultados da realidade da assistência prestada no âmbito dos cuidados espirituais.

\section{IMPLICAÇÕES PARA A PRÁTICA CLÍNICA}

Esta revisão é uma ferramenta que traz conhecimentos para os enfermeiros que desejam aprimorar os cuidados na prática sobre saúde mental (espiritualidade), voltada para os pacientes em UTI. Os achados desta revisão chamam atenção para a necessidade de disseminar e sensibilizar o cuidado espiritual entre as equipas de enfermagem. Para que isso ocorra com mais eficácia, as universidades devem atuar como facilitadoras, inserindo a abordagem do cuidado espiritual em algumas disciplinas, instruindo essa temática de forma holística e fundamentada. Assim, a espiritualidade será inserida como um cuidado vital para a saúde, sendo a saúde do indivíduo, da família e/ou comunidade.
Um papel das universidades é levar o conhecimento científico para ser aplicado na clínica, seja por meio de uma maior capacidade destas na oferta dos cursos que ministram, na realização de palestras e também na possibilidade de poderem continuar a dar aos profissionais de enfermagem uma formação continua ao longo da sua carreira activa. Os gestores também necessitam estar atentos aos fatores gerenciais de pessoal e estrutura, que possam dificultar a implementação deste e outros cuidados no ambiente de UTI.

\section{REFERÊNCIAS BIBLIOGRÁFICAS}

Azarsa, T., Davoodi, A., Markani, A. K., Gahramanian, A., \& Vargaeei, A. (2015). Spiritual wellbeing, attitude toward spiritual care and its relationship with spiritual care competence among critical care nurses. Journal of Caring Sciences, 4(4), 309. Doi: 10.15171/jcs.2015.031

Barros, A. L. B. L. D., Sanchez, C. G., Lopes, J. D. L., Dell'Acqua, M. C. Q., Lopes, M. H. B. D. M., Silva, R. D. C. G., e Ortiz, D. C. F. (2015). Processo de enfermagem: Guia para a prática. Conselho Regional de Enfermagem - SP. Disponível em: https://portal.coren-sp.gov.br/ sites/default/files/SAE-web.pdf

Brasileiro, M. D. S. E., e Brasileiro, J. E. (2017). O medo da morte enquanto mal: uma reflexão para a prática da enfermagem. Revista de Ciências Médicas, 26(2), 77-92. Doi: 10.24220/2318-0897v26n2a3582

Braz Evangelista, C., Limeira Lopes, M. E., da Costa, G., Fátima, S., de Souza Batista, P. S., Batista, V., \& de Magalhães Oliveira, A. M. (2016). Palliative care and spirituality: An integrative literature review. Revista Brasileira de Enfermagem, 69(3), 591-601. Doi: 10.1590/0034$7167.2016690324 \mathrm{i}$

Brito, F. M., Costa, I. C. P., de Andrade, C. G., de Lima, K. F. O., da Costa, S. F. G., e Lopes, M. E. L. (2013). Espiritualidade na iminência da morte: estratégia adotada para humanizar o cuidar em enfermagem. Revista Enfermagem UERJ, 21(4), 483-489. Disponível em: https:// www.e-publicacoes.uerj.br/index.php/enfermagemuerj/article/view/10013

Caldeira, S., \& Timmins, F. (2017). Implementing spiritual care interventions. Nursing Standard, 31(34), e10313. Doi: 10.7748/ns.2017.e10313 
Conselho Federal de Enfermagem (2009). Resolução COFEN no 358/2009. Sistematização da Assistência de Enfermagem e a implementação do Processo de Enfermagem em ambientes, públicos ou privados, em que ocorre o cuidado profissional de Enfermagem. Conselho Federal de Enfermagem. Disponível em: http://www. cofen.gov.br/resoluo-cofen-3582009_4384.html

Conselho Federal de Enfermagem (2017). Resolução COFEN n 564/2017. Aprova o novo Código de Ética dos Profissionais de Enfermagem. Conselho Federal de Enfermagem. Disponível em: http://www.cofen.gov.br/ resolucao-cofen-no-5642017_59145.html

Cooper, H. M. (1984). The integrative research review: A systematic approach. Beverly Hills, CA: Sage.

Dezorzi, L. W., e Crossetti, M. D. G. O. (2008). A espiritualidade no cuidado de si para profissionais de enfermagem em terapia intensiva. Revista Latino-Americana de Enfermagem, 16(2), 212-217. Doi: 10.1590/S010411692008000200007

Gulini, J. E. H. M. D., Nascimento, E. R. P. D., Moritz, R. D., Rosa, L. M. D., Silveira, N. R., \& Vargas, M. A. D. O. (2017). Intensive care unit team perception of palliative care: the discourse of the collective subject. Revista da Escola de Enfermagem da USP, 51, e03221. Doi: 10.1590/S1980-220X2016041703221

Ho, J. Q., Nguyen, C. D., Lopes, R., Ezeji-Okoye, S. C., \& Kuschner,W.G. (2018).Spiritual care in the intensive care unit: a narrative review. Journal of Intensive Care Medicine, 33(5), 279-287. Doi: 10.1177/0885066617712677

Ienne, A., Fernandes, R. A. Q., e Puggina, A. C. (2018). A espiritualidade de enfermeiros assistenciais interfere no registro do diagnóstico sofrimento espiritual?. Escola Anna Nery, 22(1), e20170082. Doi: 10.1590/2177-9465ean-2017-0082

Júnior, J. B., de Sousa Barbosa, R., de Luna Neto, R. T., Tavares, N. B. F., de Freitas, K. M., Cruz, C. A., e dos Santos Moura, Ú. H. (2015). Saúde e Espiritualidade: As narrativas profissionais na estratégia saúde da família. Id On Line Revista Multidisciplinar e de Psicologia, 9(26), 71-82. Doi: 10.14295/idonline.v9i26.329

Leoni-Scheiber, C., Mayer, H., \& Müller-Staub, M. (2020). Relationships between the Advanced Nursing Process quality and nurses' and patient' characteristics: A cross-sectional study. Nursing Open, 7(1), 419-429. Doi: 10.1002/nop2.405
Lundberg, P. C., \& Kerdonfag, P. (2010). Spiritual care provided by Thai nurses in intensive care units. Journal of Clinical Nursing, 19(7-8), 1121-1128. Doi: 10.1111/ j.1365-2702.2009.03072.x

Moreira-Almeida, A., e Lucchetti, G. (2016). Panorama das pesquisas em ciência, saúde e espiritualidade. Ciência e Cultura, 68(1), 54-57. Doi: 10.21800/231766602016000100016

Moura, A., Lunardi, R., Volpato, R., Nascimento, V., Bassos, T., e Lemes, A. (2018). Fatores associados à ansiedade entre profissionais da atenção básica. Revista Portuguesa de Enfermagem de Saúde Mental, 19, 17-26. Doi: 10.19131/rpesm.0198

Salimena, A. M. D. O., Elias, E. A., Souza, I. E. D. O., e Vieira, L. B. (2016). Falatório e ocupação no cotidiano das profissionais de enfermagem no cuidado de si e do outro. Revista Baiana de Enfermagem, 30(1), 316-324. Doi: $10.18471 /$ rbe.v1i1.14393

Silva, J. P., Garanhani, M. L., e Peres, A. M. (2015). Sistematização da assistência de enfermagem na graduação: um olhar sob o pensamento complexo. Revista Latino-Americana de Enfermagem, 23(1), 59-66. Doi: 10.1590/0104-1169.0096.2525

Sousa, P. H. G. D., Avelino, F. V. D. S. D., Andrade, E. M. L. R., Luz, M. H. B. A., \& Carvalho, N. A. R. (2018). Diagnósticos enfermeros en la unidad de Cuidados intensivos: revisión integrativa. Cultura de los Cuidados, 22(52), 223-231.

Timmins, F., \& Caldeira, S. (2017). Understanding spirituality and spiritual care in nursing. Nursing Standard, 31(22), e10311. Doi: 10.7748/ns.2017.e10311

Vale,C.C.S.O., e Líbero,A.C.A.(2017).A espiritualidade que habita o CTI. Mental, 11(21), 321-338. Disponível em: http://pepsic.bvsalud.org/scielo.php?script=sci_art text\&pid=S1679-44272017000200003

Valente, T. C., Quelho, C. T., Cavalcanti, A. P. R., e Carmo, H. (2016). Espiritualidade, religiosidade e saúde: velhos debates, novas perspectivas. Interações, 11(20), 85-97. Doi: 10.5752/P.1983-2478.2016v11n20p85

Viana, M. R. P., Silva, I. M. B., Ferreira, T. R. S., Amorim, F. C. M., e de Oliveira Soares, É. (2018). A operacionalização do processo de cuidar em enfermagem em uma unidade de terapia intensiva materna. Revista de Pesquisa: Cuidado é Fundamental, 10(3), 696-703. 